\title{
Diversidad de expectativas de los extensionistas rurales Latinoamericanos sobre la Psicología
}

F. Landini. Universidad de la Cuenca del Plata.

\begin{abstract}
Resumen
La psicología posee potencialidad para contribuir con la extensión rural. Sin embargo, las características de su rol, así como las necesidades específicas en diversos países aún no han sido clarificadas. Buscando analizar de manera diferencial las expectativas que extensionistas rurales latinoamericanos de 12 países tienen sobre la psicología, se realizó una encuesta. De los 654 extensionistas encuestados, 585 consideraron que la psicología podría contribuir a su trabajo. Se concluye que las expectativas en los diferentes países son diversas, aunque poseen elementos compartidos. Existen dos tipos de expectativas: aquellas como la gestión de grupos y procesos participativos con productores y la capacitación y asesoramiento de extensionistas, que pueden ser utilizadas para guiar el trabajo de los psicólogos; y aquellas que tienden a poner a los productores en el lugar de objetos de intervención y no de sujetos de cambio, las cuales necesitan ser puestas en discusión junto con los extensionistas.
\end{abstract}

Palabras clave: ambiente rural; expectativas del rol; desarrollo económico; América Latina.

\begin{abstract}
Diversity of expectations of Latin American rural extensionists of Psychology. Psychology has potential to contribute to rural extension. Nonetheless, the characteristics of its role, as well as the specific needs in different countries have not been yet clarified. Thus, aiming at analyzing Latin American rural extensionists' differential expectations of psychology in 12 countries, a survey was conducted. Of 654 surveyed extensionists, 585 considered psychology could contribute to their work. Expectations from psychology in the different countries are diverse, despite the fact some of them are shared. There are two different types of expectations: those such as the management of farmers' groups and participatory processes, and the training and advising of extensionists, which can be used to guide psychologists' practices; and those that place farmers in the position of objects of psychologists' interventions and not of subjects of transformation, which has to be put up for discussion with extensionists.
\end{abstract}

Keywords: rural environments; role expectations; economic development; Latin America.

\section{Resumo}

Diversidade de expectativas dos extensionistas rurais da América Latina sobre a Psicologia. A psicologia tem a potencialidade de contribuir com a extensão rural. Não obstante, as características de seu papel, assim como as necessidades específicas em diversos países, ainda não têm sido esclarecidas. Procurando analisar de maneira diferencial as expectativas que os extensionistas rurais de 12 países latinoamericanos têm sobre a psicologia, a enquete foi implementada. Dos 654 extensionistas rurais enquetados, 585 disseram que a psicologia poderia contribuir com seu trabalho. Conclui-se que as expectativas nos diferentes países são diversas, mas têm elementos compartilhados. Existem dois tipos de expectativas: aquelas como a gestão de grupos e processos participativos com os agricultores e a capacitação e o assessoramento dos extensionistas, as quais podem ser utilizadas para guiar os trabalhos dos psicólogos; e aquelas que tendem a pôr aos agricultores no lugar de objetos de intervenção e não de sujeitos de transformação, as quais têm que ser postas em discussão junto aos extensionistas.

Palavras-chave: ambientes rurais; expectativas do papel; desenvolvimento econômico; psicólogos; América Latina. 
En América Latina, según el Banco Mundial, en el año 2015 el $20 \%$ de la población vivía en áreas rurales, lo que representaba más de 127 millones de personas ${ }^{1}$. Por su parte, se observa que tanto a nivel latinoamericano como mundial los niveles de pobreza y de pobreza extrema son más altos en ámbitos rurales que en urbanos (Organización de las Naciones Unidas para la Alimentación y la Agricultura - FAO, 2015; Vakis, Rigolini \& Lucchetti, 2015), lo que justifica una visión particularmente atenta a lo que sucede en los primeros. Dentro de las poblaciones rurales, los agricultores familiares aparecen como un actor fundamental para mejorar los índices de seguridad alimentaria, empleo y pobreza (Comisión Económica para América Latina y el Caribe, (Comisión Económica para América Latina y el Caribe - CEPAL, Instituto Interamericano de Cooperación para la Agricultura - IICA, \& Organización de las Naciones Unidas para la Alimentación y la Agricultura - FAO, 2013). No obstante, en América Latina la agricultura familiar enfrenta hoy grandes desafíos, dentro de los que pueden mencionarse la presión del agronegocio y la degradación de los recursos naturales.

En este contexto, la extensión rural se destaca como una estrategia intervención orientada a impulsar procesos de desarrollo rural, con especial foco en la agricultura familiar (Ardila, 2010). Si bien la extensión rural ha sido definida históricamente de diferentes maneras (Leeuwis, 2004), actualmente se la considera como el conjunto de las diferentes actividades orientadas a proporcionar la información y los servicios que tanto los agricultores como otros actores del sector rural necesitan para desarrollar sus prácticas productivas, organizativas, comerciales o de gestión (Christoplos, 2010). Tradicionalmente, la extensión rural ha estado monopolizada por profesionales de las ciencias agrarias (Landini \& Bianqui, 2014). No obstante, el progresivo reconocimiento de los factores grupales, institucionales y sociales implicados en los procesos de innovación y desarrollo (Leeuwis, 2004; Leeuwis \& Aarts, 2011; Sulaiman \& Davis, 2012) muestra la importancia de un mayor involucramiento en ellos de las ciencias sociales en general (Carballo, 2002) y de la psicología en particular (Landini, Murtagh, \& Lacanna, 2009).

Si bien desde mediados del siglo XX la psicología ha contribuido de cierta manera al estudio de los procesos de comunicación y de adopción de tecnologías vinculados con la extensión rural, su aporte a la comprensión de la dimensión psicosocial de la extensión y el desarrollo rural ha sido en general pobre, tanto por la escasez cuantitativa de sus contribuciones como por la limitación de sus abordajes (Landini, 2015a; Murtagh \& Landini, 2011). No obstante, más recientemente, en América Latina se observa un creciente interés por el estudio psicosocial de los procesos de extensión y desarrollo rural (Bianqui, Mathot, Vázquez, \& Landini, 2015; Landini, Leeuwis, Long \& Murtagh, 2014; Landini, Long, Leeuwis, \& Murtagh, 2014). En esta línea, el autor de este trabajo ha llevado adelante un estudio orientado a clarificar las contribuciones de la psicología a este campo analizando las expectativas que los extensionistas rurales de 12 países latinoamericanos tienen sobre la psicología y los psicólogos. En concreto, se trata de un estudio llevado adelante entre 2010 y 2014 en Argentina, Bolivia, Brasil, Chile, Costa Rica, Cuba, Ecuador, El Salvador, México, Paraguay, Perú y Uruguay.

En un primer trabajo, este estudio permitió identificar como principales expectativas de los extensionistas recibir apoyo de los profesionales de la psicología para (a) el manejo de procesos grupales y participativos con productores y para (b) la transferencia de tecnologías y el cambio de la mentalidad de los productores. A esto se une la expectativa de (c) recibir capacitación y herramientas prácticas para enfrentar los problemas de su práctica que exceden su competencia técnica (Landini, 2015b). A la vez, este estudio latinoamericano también dio pie a la identificación de extensionistas con diferentes perfiles de expectativas sobre la psicología, encontrándose tres grupos diferenciados (Landini, en prensa). Por un lado, extensionistas que mencionan más frecuentemente la potencialidad de la psicología para trabajar directamente con los extensionistas, pero menos la posibilidad de hacerlo directamente con productores. El segundo grupo, a la inversa, destaca la potencialidad de los psicólogos para trabajar con grupos de productores y con procesos participativos. Finalmente, el tercero tiende a tomar a los productores como objetos pasivos de la intervención de la psicología, destacándose con menos frecuencia la posibilidad generar abordajes interdisciplinarios.

No obstante, pese a la diversidad existente a nivel latinoamericano en lo que hace a climas, culturas y matrices productivas, estos trabajos previos sólo se han ocupado de analizar las expectativas de los extensionistas rurales a nivel regional, pero no por países específicos. Así, las diferencias entre ellos han quedado invisibilizadas, lo que redujo la utilidad de estos resultados para los investigadores y los profesionales que trabajan en ellos. En consecuencia, en el presente trabajo se propone un re-análisis de la información presentada 
en los trabajos previos con el fin de describir y analizar de manera diferenciada las expectativas sobre la psicología de los extensionistas en los países estudiados.

\section{Método}

Se realizó una investigación cuali-cuantitativa de tipo transversal a partir de una encuesta a extensionistas rurales de 12 países latinoamericanos. Las encuestas fueron enviadas y recibidas por e-mail con apoyo de autoridades de extensión rural de los diferentes países. En total se obtuvieron 654 respuestas (Argentina: $n=220$; Bolivia: $n=19$; Brasil: $n=52$; Chile: $n=41$; Costa Rica: $n$ = 32; Cuba: $n=33$; Ecuador: $n=74$; El Salvador: $n=34$; México: $n=60$; Paraguay: $n=26$; Perú: $n=31$; Uruguay: $n=32$ ). Si bien originalmente se apuntó a obtener al menos 30 encuestas de cada país, la diferencia a nivel de las muestras se relaciona con el apoyo obtenido en cada uno de ellos.

Las instituciones que colaboraron con la recolección de la muestra fueron seleccionadas en cada país priorizando aquellas de carácter nacional, generalmente públicas. Cuando estas no existían o no fue posible conseguir su colaboración, se pidió apoyo a instituciones estaduales (como en el caso de Brasil y Ecuador) o a diferentes ONGs que llevaban adelante acciones de extensión en diferentes territorios (como en Bolivia y Perú).

Como criterio de inclusión en la muestra se estableció que quienes respondían tenían que estar trabajando en la actualidad como extensionistas rurales, trabajar o haber trabajado con pequeños productores como extensionistas y tener al menos 1 año de experiencia en tareas de extensión. No se estableció una definición general de extensión rural ni de pequeño productor, sino que se pidió a las instituciones de extensión rural que colaboraron con la investigación que utilizaran sus propias definiciones de estos conceptos.

A la vez, dado que no se disponía de datos consistentes sobre la cantidad de extensionistas en cada país o sobre su distribución por sexo y edad, se optó por no establecer cuotas según estas variables. Por su parte, en lo referido a la distribución por región, tampoco se establecieron cuotas, aunque en este caso no sólo por la falta de información sobre la distribución de extensionistas a nivel nacional, sino especialmente por la limitación operativa de estar trabajando al mismo tiempo con 12 países y de no poder acceder directamente a las muestras. De todas maneras, sí se pidió a las instituciones colaboradoras que hicieran lo posible por que la muestra estuviera equilibrada territorialmente.
Atendiendo al modo en que las muestras fueron obtenidas en cada país, debe tenerse en claro que ni los resultados del presente trabajo son generalizables en un sentido estadístico a América Latina ni las muestras nacionales representan al país en el que fueron obtenidas. Así, en un sentido estricto, los resultados de esta investigación sólo aplican a las muestras a las que refieren, lo que hace evidente la necesidad de conducir estudios de nivel nacional estadísticamente representativos y de mayor profundidad. En cualquier caso, esto no quita el valor heurístico de los datos en un contexto en el cual se carece de información sobre los temas abordados.

La encuesta utilizada fue construida por el equipo que llevó adelante la investigación. Las diferentes preguntas fueron incluidas para alcanzar los objetivos propuestos. Para chequear la comprensión de la redacción de las preguntas, se realizó una prueba piloto con un grupo reducido de extensionistas argentinos, lo que llevó a ajustes mínimos. A la vez, dado que las palabras pueden tener diferente sentido según los países, al aplicar la encuesta en cada uno de ellos se pidió a un investigador o extensionista local que revisara el instrumento y sugiriera modificaciones relativas a la comprensión.

La encuesta contenía preguntas socio-demográficas cerradas y preguntas abiertas referidas a dos temas diferentes: por un lado, los problemas enfrentados en el trabajo de extensión rural; por el otro, las contribuciones potenciales de la psicología para enfrentarlos. Entre ambos grupos de preguntas abiertas se incluía la siguiente: "De los problemas y dificultades mencionados en las preguntas anteriores, ¿usted piensa que la psicología podría ayudar a resolver alguno de ellos?" La respuesta era dicotómica, con opciones de respuesta Sí/No. En total, 585 extensionistas respondieron afirmativamente, mientras que 64 lo hicieron de manera negativa y 3 no respondieron.

Con el fin de analizar las expectativas de los extensionistas rurales encuestados sobre la psicología, se trabajó solamente con quienes habían respondido de manera afirmativa. A nivel operativo, se definen como expectativas de los extensionistas sobre la psicología a aquellas afirmaciones de los encuestados en las cuales estos describen las actividades o acciones que los psicólogos podrían llevar adelante en el contexto de la extensión rural. Desde la perspectiva metodológica, las expectativas de los extensionistas sobre la psicología constituyen el tema de investigación. A la vez, las distintas expectativas que aparecen en las encuestas constituyen las variables de la investigación, siendo sus 
valores posibles presencia o ausencia. En concreto, se define como presente una variable cuando la expectativa que expresa ha sido mencionada en una encuesta, y ausente cuando esto no ha sucedido. Atendiendo al carácter descriptivo de la investigación y a que no se busca pronosticar ninguna distribución de las expectativas de los extensionistas sobre la psicología, no se parte de ninguna hipótesis a priori (Hernández Sampieri, Fernández Collado, \& Baptista Lucio, 2010).

Para identificar las expectativas de los encuestados sobre la psicología se siguieron los principios generales de la Teoría Fundamentada (Giraldo, 2011). En este caso, sólo se toman en cuenta las respuestas a las preguntas específicas sobre las contribuciones potenciales de la psicología al trabajo de extensión. Luego de leer las respuestas, con el apoyo del software Atlas Ti se construyeron categorías para marcar fragmentos de texto con afirmaciones referidas a expectativas semejantes, siguiendo una estrategia inductiva. Este proceso permitió construir un listado de categorías (es decir, de expectativas sobre la psicología). A continuación, se construyó una definición para cada categoría, la que fue utilizada para marcar todas las encuestas. En este proceso, surgieron dudas sobre las categorías a las que correspondían determinadas porciones de texto. Esto llevó a la necesidad de realizar ajustes en las categorías y a incorporar en las definiciones criterios para incluir o excluir fragmentos que presentaran dudas. Así, con estas definiciones finales se procedió a categorizar de manera definitiva todo el material. Tomando estos resultados, en este artículo se presentan, de manera diferenciada por países, aquellas expectativas sobre la psicología mencionadas de manera más frecuente. En este sentido, se toman aquellas mencionadas en al menos un país por un $25 \%$ de sus encuestados, y se excluyen las que no se corresponden específicamente con expectativas.

En segundo lugar, también se buscó identificar conjuntos de expectativas sobre la psicología que tendían a aparecer agrupadas. Para esto se llevó a cabo un análisis de conglomerados bi-etápico con el apoyo del software SPSS, dada la potencialidad del procedimiento para identificar agrupaciones dentro de un conjunto de datos que a simple vista podrían no detectarse (Pérez, 2001). Las variables utilizadas para el análisis de conglomerados fueron 12. Estas incluyeron las 9 expectativas mencionadas por al menos un promedio del $20 \%$ de los extensionistas de cada país, a las cuales se sumaron tres categorías adicionales, también surgidas del análisis cualitativo de las encuestas: si los extensionistas proponían o no que los psicólogos trabajaron con productores, con los propios extensionistas, o a un nivel institucional o supra-institucional. El análisis permitió arribar a una solución de tres conglomerados (para más detalles ver Landini, en prensa). En los resultados se presenta y analiza la distribución por conglomerado en cada país.

\section{Resultados}

Fueron 13 las expectativas sobre la psicología mencionadas por los encuestados que estaban presentes al menos en un $25 \%$ de las encuestas de un país. El análisis estadístico realizado mostró que todas ellas se distribuyen de manera desigual entre los países, lo que indica que tienen potencialidad para detectar diferencias entre ellos. En todos los casos, utilizando $\chi^{2}, p \leq 0,016$.

En la Tabla 1 se presentan las expectativas de los extensionistas que consideraron que la psicología podía contribuir con su trabajo, incluyendo nada más que aquellas mencionadas por al menos el $25 \%$ de los encuestados de cada país. Por su parte, en la Tabla 2 se muestra de qué manera estas expectativas se distribuyen de manera desigual en cada país, mencionándose en cada uno de ellos sólo aquellas que han sido indicadas por al menos el $25 \%$ de los integrantes de la muestra nacional.

En la Tabla 3 se presenta la distribución porcentual de extensionistas correspondientes a cada uno de los conglomerados identificados, según país.

Partiendo de los datos presentados en la Tabla 3 , se analizó la relación entre la distribución de los conglomerados y los países, obteniéndose un $\chi^{2}=114,6$ (22) y un valor $p<0,001$, por lo que se concluye que esta variable también resulta útil para diferenciar entre los distintos países estudiados.

\section{Discusión}

A nivel general, se observa que las expectativas de los extensionistas, así como las tipologías de expectativas construidas, se distribuyen de manera diferente en los distintos países, lo que permite concluir que resulta relevante identificar las expectativas específicas en cada uno de ellos en lugar de tomar resultados genéricos de nivel latinoamericano. Esto resulta particularmente útil para quien tiene que trabajar en contextos específicos, ya que permite prever de manera más precisa el tipo de expectativas con las que puede encontrarse.

Los resultados de la muestra argentina resultan particularmente interesantes para los psicólogos con intenciones de trabajar en el contexto de la extensión 
Tabla 1. Expectativas de los Extensionistas Rurales Sobre la Psicología en América Latina.

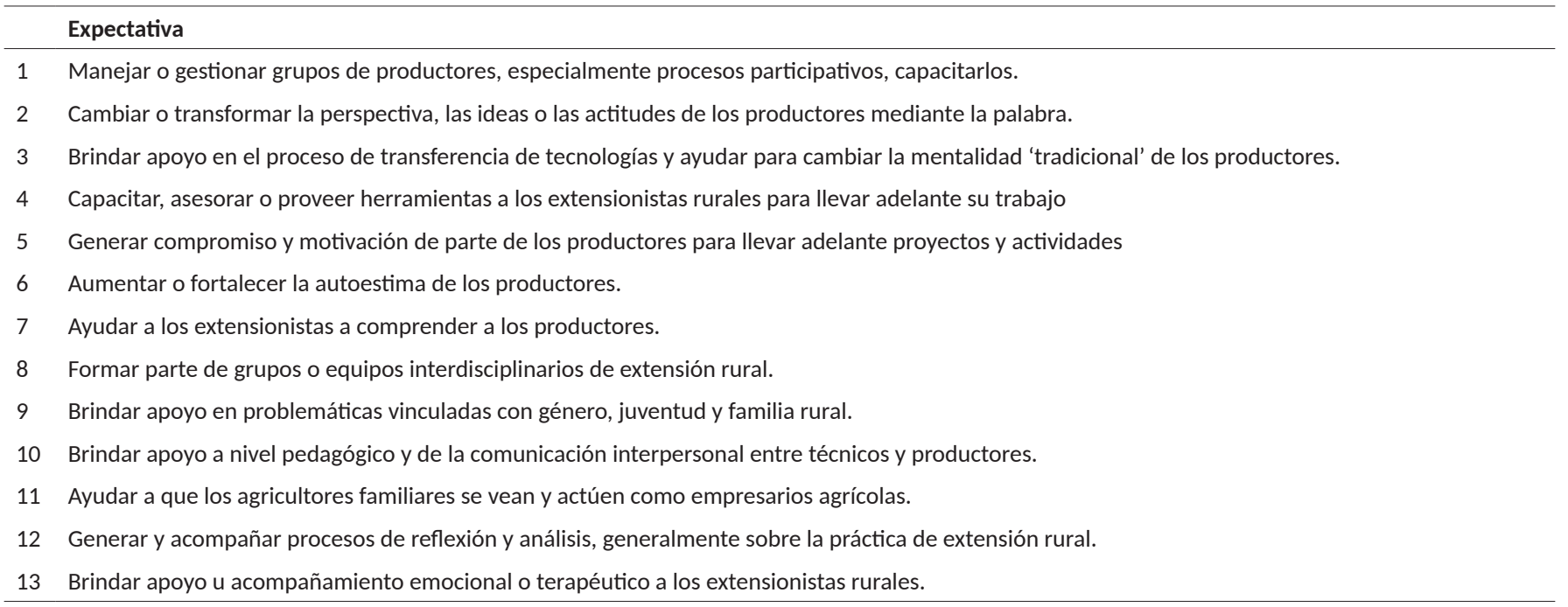

rural. En primer lugar, se observa un equilibrio entre las expectativas de que los psicólogos trabajen con productores y con extensionistas, lo que amplía las posibilidades de acción. A la vez, se destaca un porcentaje comparativamente alto (del $41 \%$ ) de extensionistas que mencionan la posibilidad de que los psicólogos trabajen como parte de equipos interdisciplinarios de extensión rural, lo que constituye una importante base para proponer una forma específica de inserción en las instituciones de extensión.

A los fines de facilitar el análisis de la distribución por países de los tres tipos diferenciados de expectativas de los extensionistas sobre la psicología surgidos del análisis de conglomerados a nivel latinoamericano que fueron mencionaron en los antecedentes (para más detalles véase Landini, en prensa), a continuación se amplía la descripción de las características de cada uno de ellos. En concreto, en dicho trabajo se observó que los extensionistas que correspondían al primer conglomerado se caracterizaban por destacar la necesidad de que los psicólogos ayuden a entender a los productores, trabajen directamente con los extensionistas y formen parte de equipos interdisciplinarios. Respecto del segundo, se observó que una preferencia clara de los extensionistas que los psicólogos trabajen con grupos de productores e implementen procesos participativos, en lugar de trabajar apoyándolos o asesorándolos a ellos como técnicos. Finalmente, se observó que el tercer conglomerado se caracterizaba por extensionistas que esperan que los psicólogos trabajen de manera directa con los productores y ayuden a persuadirlos para que adopten tecnologías, tomándolos casi como objetos de intervención en lugar de cómo sujetos con los cuales establecer un vínculo horizontal o de diálogo.
Respecto del caso de Argentina, el presente artículo muestra un predominio de extensionistas del conglomerado 1. Esto implica que se trata de extensionistas que incorporan una mirada más abierta de su práctica, lo que facilita que puedan reflexionar sobre su propio rol en la extensión y sobre las posibilidades de la psicología para trabajar directamente con ellos (y no únicamente con los productores). A la vez, se observa una muy baja presencia de extensionistas correspondientes al conglomerado 3 , es decir, con tendencia a pensar a los productores como objetos sobre los que la psicología debe intervenir para cambiar sus modos de producir y de pensar, lo que resulta favorable.

Si bien no pueden establecerse relaciones lineales, este perfil parece asociarse bien con el nuevo enfoque propuesto por la Instituto Nacional de Tecnología Agropecuaria (INTA), institución referente en Argentina en lo que hace a extensión rural. En efecto, en el Plan Estratégico Institucional 2005-2015 (INTA, 2004), el INTA destaca la necesidad de recuperar tanto la complejidad territorial como la social de los procesos de innovación y extensión rural, lo que sostiene la necesidad de pensar en abordajes interdisciplinarios, alejándose así de la tendencia a ver a los productores como objetos, más asociada a una visión tradicional de la extensión rural (Landini, 2016).

En Bolivia, el perfil de expectativas parece hasta cierto punto contrastante. Por un lado, más del $50 \%$ de los extensionistas se ubican en el conglomerado 1 , lo que implica que dan gran importancia a la capacitación y orientación que podrían recibir de parte de los psicólogos. Esto se complementa con el porcentaje más alto de expectativas vinculadas con ayudar a comprender al productor (44\%), lo que es consistente con el carácter 
Diversidad de expectativas de los extensionistas rurales

Tabla 2. Expectativas de los Extensionistas Rurales Sobre la Psicología en los Distintos Países.

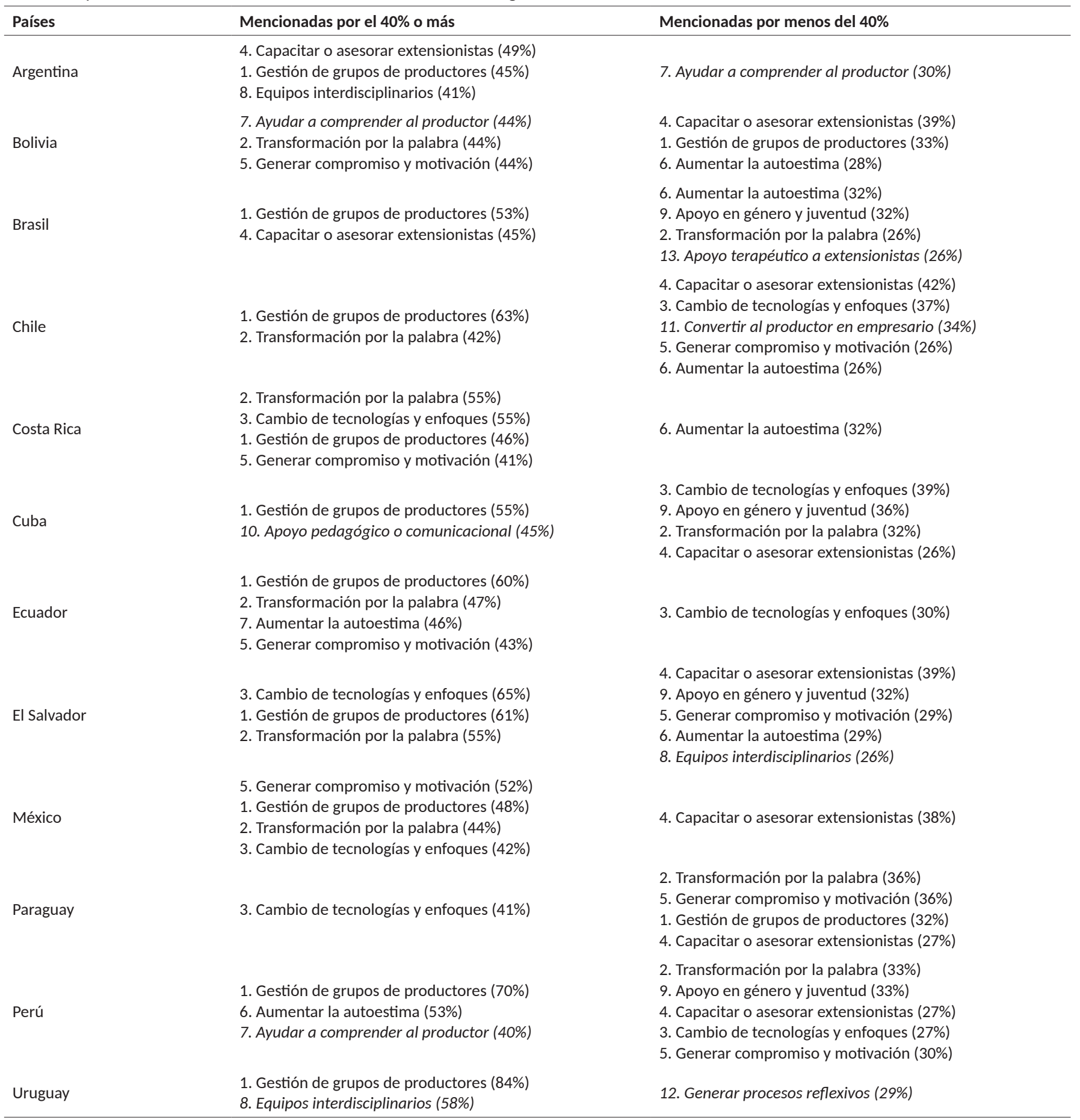

En cursiva se indican aquellas expectativas que alcanzaron el $25 \%$ en tres o menos países.

plurinacional de Bolivia y su elevado porcentaje de población indígena, portadora de prácticas comunitarias y culturales propias (Quispe \& Delgado, 2010). No obstante, por otra parte, también se observa un importante porcentaje de extensionistas en el conglomerado 3 , los cuales tienden a ubicar a los productores en el lugar de objetos de intervención de la psicología. Este contraste parece tener de base la contraposición entre un esquema de extensión más tradicional, asociado a la transferencia de tecnologías y a la búsqueda de un aumento en la productividad, y otro más de tipo comunitario, apoyado en el diálogo de saberes.

Este contexto lleva a pensar cómo deberían actuar los psicólogos frente a expectativas que ponen a los 
Tabla 3. Conglomerados de Expectativas Sobre la Psicología por País.

\begin{tabular}{lccc}
\hline Países & Conglomerado 1 & Conglomerado 2 & Conglomerado 3 \\
\hline Argentina & $59,59 \%$ & $33,16 \%$ & $7,25 \%$ \\
Bolivia & $55,56 \%$ & $11,11 \%$ & $33,33 \%$ \\
Brasil & $42,55 \%$ & $29,79 \%$ & $27,66 \%$ \\
Chile & $28,95 \%$ & $28,95 \%$ & $42,11 \%$ \\
Costa Rica & $22,73 \%$ & $18,18 \%$ & $59,09 \%$ \\
Cuba & $45,16 \%$ & $16,13 \%$ & $38,71 \%$ \\
Ecuador & $40,00 \%$ & $27,14 \%$ & $32,86 \%$ \\
El Salvador & $25,81 \%$ & $19,35 \%$ & $54,84 \%$ \\
México & $28,85 \%$ & $19,23 \%$ & $51,92 \%$ \\
Paraguay & $36,36 \%$ & $13,64 \%$ & $50,00 \%$ \\
Perú & $40,00 \%$ & $33,33 \%$ & $26,67 \%$ \\
Uruguay & $32,26 \%$ & $61,29 \%$ & $6,45 \%$ \\
Promedio & $43,76 \%$ & $28,55 \%$ & $27,69 \%$ \\
\hline
\end{tabular}

productores en el lugar de objetos de intervención, ya que responder a ellas implicaría establecer un vínculo que tiende a negar su valor como sujetos. Así, si bien habrá que decidir contextualmente de qué manera conducirse, parece resultar prioritario poner en discusión estas expectativas con los propios extensionistas, con el fin de construir juntos estrategias de trabajo superadoras (Landini, 2015b).

El caso de Brasil tiene algunas similitudes con el de Argentina, especialmente respecto al equilibrio entre expectativas de que los psicólogos trabajen tanto con productores como con extensionistas. No obstante, en este caso se observa un porcentaje más alto de extensionistas en el conglomerado 3. Un elemento a destacar en el caso de Brasil es la expectativa de que los psicólogos brinden apoyo terapéutico a los extensionistas (26\%), algo que no aparece en ninguno de los otros países con este nivel de importancia, pero que podría estar sugiriendo una necesidad específica que debería ser tomada en cuenta por aquellos psicólogos interesados en trabajar en extensión rural en el contexto brasileño.

Los resultados de la muestra chilena evidencian un predominio de expectativas orientadas al trabajo con productores a partir de la gestión de grupos y de procesos participativos (63\%). Esto se complementa con un alto porcentaje de extensionistas ubicados en el conglomerados $3(42 \%)$ y con altos porcentajes de expectativas que destacan la 'potencialidad' de la psicología para actuar sobre los productores, transformándolos por medio de la palabra (42\%) y contribuyendo al proceso de adopción de tecnologías y de cambio de su mentalidad (37\%). Esto representa un llamado de atención para los psicólogos que quieran trabajar en este contexto, ya que parte de su trabajo será, como se señaló anteriormente, poner en discusión estas expectativas y los supuestos que las guían, para ayudar a pensar otros modelos de práctica. Al mismo tiempo, también se percibe la fuerte internalización de la lógica neoliberal en la sociedad chilena (Gómez Leyton, 2008), al mencionarse con un grado alto de frecuencia la expectativa de que los psicólogos ayuden a convertir a los productores en empresarios (34\%), cuando en ningún otro de los países estudiados esta expectativa supera el $25 \%$.

Se observan importantes similitudes entre el caso chileno y el costarricense. En Costa Rica se observa que el $59 \%$ de los encuestados se ubican en el conglomerado 3 , siendo éste el porcentaje más alto de los 12 países latinoamericanos estudiados. En línea con esto, aparecen expectativas vinculadas con la posibilidad de que los psicólogos trabajen con los productores (y no con los propios extensionistas), lo que refuerza la idea de poner a los productores en el lugar de objetos de intervención y no de sujetos de promoción y desarrollo. En concreto, se mencionan en un 55\% de las encuestas analizadas las expectativas de que los psicólogos ayuden en el proceso de transferencia de tecnologías y de cambio de mentalidad de los productores y que aporten a su transformación por medio de la palabra. Nuevamente, aquí se destaca la importancia de que los psicólogos que elijan desempeñarse en este campo en Costa Rica puedan poner en discusión estas expectativas y supuestos, con el fin de encontrar alternativas de acción que puedan orientarse a la promoción y al desarrollo de capacidades de los agricultores familiares.

Por su parte, el caso cubano presenta un perfil contrastado. Por un lado, un $45 \%$ de los encuestados poseen expectativas asociadas al conglomerado 1, posiblemente vinculadas con el alto porcentaje de referencias a la potencialidad de la psicología para brindar apoyo en el ámbito pedagógico o comunicacional. Por el otro, un $39 \%$ de los extensionistas se encuentran ubicados en el conglomerado 3 y poseen expectativas vinculadas con recibir apoyo para cambiar la mentalidad de los productores (39\%). La expectativa vinculada con el apoyo pedagógico/comunicacional resulta particularmente significativa, ya que es mencionada por el $45 \%$ de los encuestados cubanos, siendo Cuba el único país donde el porcentaje supera el $25 \%$, lo que implica que los psicólogos trabajando en extensión rural en Cuba tendrán que prepararse especialmente para contribuir en este 
sentido. A la vez, también se destaca la expectativa de recibir por parte de los psicólogos apoyo en el ámbito de género, juventud y familia (36\%), siendo el porcentaje más alto comparado con el resto de los países estudiados. Finalmente, dada la alta incidencia de extensionistas ubicados en el conglomerado 3 , también habrá que tener presente la importancia de poner en discusión las expectativas y supuestos que subyacen a él cuando resulte posible.

La muestra ecuatoriana presenta un equilibrio cuantitativo entre los 3 conglomerados, aunque un 33\% de la muestra nacional se encuentra asociada al conglomerado 3, con las implicaciones que esto conlleva. Adicionalmente, se destaca un dato interesante, que es que el tercer conglomerado cuenta con un $48 \%$ de mujeres, frente a un $18 \%$ y a un $16 \%$ en los otros dos, lo que sugiere que las extensionistas mujeres pueden tener mayor tendencia a ubicar a los productores en el rol de objetos de intervención de la psicología que los hombres. Por otra parte, a nivel general, se observa un alto porcentaje de encuestados que esperan que los psicólogos trabajen con grupos de productores (60\%), mientras que las expectativas de que lo hagan con extensionistas bajan al $13 \%$. Así, queda claro que existirá mayor facilidad para generar propuestas de trabajo con productores que con técnicos. Finalmente, pensando en el trabajo de los psicólogos con los productores, se destaca el interés de trabajar en el aumento de la autoestima (46\%), lo que requeriría un análisis específico de las razones (y problemáticas) que llevan a esta situación.

En El Salvador, el 55\% de los encuestados han quedado ubicados en el conglomerado 3 , en línea con el alto porcentaje de expectativas de que los psicólogos aporten al cambio productivo y de mentalidad de los productores (65\%) y contribuyan a su transformación por medio de la palabra (55\%). Así, el trabajo de los psicólogos en El Salvador en el ámbito de la extensión rural parece requerir como acción prioritaria el poner en discusión las expectativas mismas que se tienen sobre su trabajo. Ahora bien, observando el importante porcentaje de encuestados que mencionan que los psicólogos podrían capacitar y asesorar a los extensionistas rurales (39\%), se entrevé una interesante posibilidad de apertura a este diálogo, lo que debería ser considerado. Finalmente, dado que un $32 \%$ hace referencia al interés de que la psicología contribuya en temas vinculados con género, juventud y familia, se hace relevante que los profesionales interesados estén preparados para brindar su apoyo en estas cuestiones.
Los resultados del caso mexicano son similares a los de El Salvador. Se observa un importante porcentaje de extensionistas posicionados en el conglomerado 3 (52\%), lo que implica su tendencia a pensar que el rol de los psicólogos en el trabajo de extensión implicaría tomar a los productores como objetos y no como sujetos de su intervención. Esto se sostiene en altos porcentajes de expectativas vinculadas con la gestión de grupos de productores (48\%), la transformación de productores por la palabra (44\%) y el apoyo a la adopción de tecnologías y el cambio de mentalidad de los productores (42\%). En paralelo, un $38 \%$ hace referencia al interés de que los psicólogos puedan capacitarlos y brindarles su asesoramiento, espacio que puede ser utilizado para discutir expectativas y delinear acciones para aumentar el compromiso y la participación de los productores que se apoyen en un modelo diferente de vínculo, más dialógico y menos vertical (Freire, 1973).

Por su parte, el caso paraguayo se caracteriza por ser el único con sólo una expectativa mencionada por más del $40 \%$. Esto podría estar indicando un menor grado de interés en la psicología en términos generales, lo que llevaría a una mayor dificultad para que los psicólogos trabajen en este contexto. A la vez, se observa que la expectativa más mencionada se refiere a que los psicólogos contribuyan con la transferencia de tecnologías y la búsqueda de cambio de mentalidad de los productores (41\%), lo que se vincula con una concepción tradicional y difusionista de la extensión rural, un problema indicado como central en Paraguay (Landini, 2012). Por último, debe destacarse que el $50 \%$ de los extensionistas se encuentra ubicado en el conglomerado 3 , lo que debe ser tenido en cuenta al momento de planificar acciones y estrategias de dialógico con los extensionistas locales.

Analizando el caso peruano, se observan algunas similitudes con el de Bolivia, tal vez por tratarse en ambos casos de naciones andinas, particularmente en lo que hace al interés en que los psicólogos ayuden a comprender al productor, algo no tan frecuente en el resto de los países, especialmente por el porcentaje de referencias del $40 \%$. Sin dudas, esta expectativa brinda una interesante guía para plantear posibles contribuciones concretas para aportar al trabajo de extensión rural desde la psicología. Un elemento importante para destacar en la muestra peruana es que el $70 \%$ de los encuestados mencionó que los psicólogos podrían trabajar gestionando grupos de productores y procesos participativos, algo que contrasta con sólo un $27 \%$ que espera recibir capacitación y asesoramiento por parte de 
psicólogos. Así, el trabajo con productores aparece como un área de acción destacada, mientras que la alternativa de hacerlo directamente con los extensionistas puede llegar a encontrarse con mayores resistencias. Otra cuestión a mencionar es la expectativa de que los psicólogos ayuden a aumentar la autoestima de los productores, algo que parece generar particular preocupación en los encuestados (53\%). Finalmente, en lo que hace a la distribución de los encuestados en los distintos conglomerados, se observa que aquellos vinculados con el tercero son minoría (27\%). No obstante, cabe mencionar que frente a un promedio de edad de 40 años de quienes fueron clasificados como conglomerado 1 y de 46 años en relación al número 2 , aquellos asociados al 3 tienen un promedio de 31 años, lo que sugiere que habrá mayores probabilidades de encontrarse con extensionistas con las expectativas propias de este conglomerado mientras más jóvenes sean.

Finalmente, el caso de Uruguay resulta particularmente interesante. Por un lado, se observa la expectativa (con el más alto porcentaje de todas las muestras), de que los psicólogos gestionen grupos y procesos participativos con productores (84\%). A la vez, en la muestra uruguaya también se observa el porcentaje más alto de referencias a que los psicólogos podrían trabajar como parte de equipos interdisciplinarios (58\%), más si se lo contrasta con el hecho de que en nueve de los 12 países estudiados esta expectativa no llega al $25 \%$. Para comprender este escenario es necesario mencionar que la encuesta fue tomada en Uruguay cuando el Programa Uruguay Rural estaba en su apogeo, el cual se había propuesto trabajar de manera articulada factores sociales y productivos en el contexto de organizaciones de productores (Moraes, Oreggioni, \& Picos, 2010), lo que llevó incluso a la incorporación de psicólogos al trabajo de extensión rural (Landini \& Riet, 2015). Así, en ese contexto, resultaba claro que el rol de los profesionales de las ciencias sociales era trabajar con iniciativas asociativas en el marco de equipos interdisciplinarios. Por su parte, el análisis de la distribución por conglomerados muestra un muy bajo porcentaje de extensionistas categorizados dentro del tercero (6\%), mientras que la mayoría queda ubicado en el segundo (61\%). Así, se observa un predominio de expectativas vinculadas con el trabajo de los psicólogos con productores, especialmente en cuestiones vinculadas con lo asociativo o participativo, pero con pocas referencias a la posibilidad de brindar capacitación o asesoramiento a extensionistas. De esta forma, la participación de psicólogos en el trabajo de extensión rural aparece claramente legitimada en lo que se refiere al trabajo con productores, aunque posiblemente requeriría un trabajo o esfuerzo adicional abrir la posibilidad de hacerlo como asesor o capacitador de extensionistas.

\section{Conclusiones}

El presente trabajo ha permitido evidenciar que las expectativas que tienen los extensionistas rurales de muestras de 12 países latinoamericanos sobre la psicología no son homogéneas, por lo que resulta relevante un análisis de aquellas propias de cada contexto, si lo que se busca es dar forma el rol profesional de la psicología en cada uno de ellos. No obstante, como se señaló anteriormente, el objetivo primario de conocer estas expectativas no es prepararse para seguirlas al pie de la letra sino, más bien, diseñar estrategias de trabajo que tengan mayor potencialidad de ser aceptadas y de generar impactos en términos de procesos de desarrollo rural.

Las expectativas identificadas, a grandes rasgos, parecerían poder ser diferenciadas en dos grupos. Primero, aquellas sobre las que no existe ningún reparo específico para establecerlas como focos de la intervención, como ser la gestión de grupos y procesos participativos con productores, la capacitación y asesoramiento de extensionistas, el aumento de la autoestima de los productores, el apoyo pedagógico y comunicacional, y el trabajo en el área de género, juventud y familia, entre otros. Teniendo en cuenta que se trata de necesidades sentidas de los extensionistas, parece resultar valioso, tanto por cuestiones estratégicas como de utilidad social, generar acciones específicas para formarse y responder a ellas como psicólogos. A la vez, también existe un segundo grupo de expectativas, relacionadas con recibir apoyo para la transferencia de tecnologías y el cambio de mentalidad de los productores, y con la potencialidad de la psicología para generar transformaciones en las personas por medio de la palabra, sobre las que existen ciertos reparos. Concretamente, la tendencia a poner a los productores en el lugar de objetos pasivos de la intervención de la psicología, y no de sujetos portadores de recursos y potencialidades que deben ser desarrolladas, como propone la psicología comunitaria. Respecto de estas expectativas, el rol propuesto para los psicólogos interesados en el trabajo de extensión no será acatarlas sino, por el contrario, ponerlas como tema de discusión y reflexión, con el fin repensar los supuestos a partir de los cuales se construyen y generar así alternativas superadoras. 
Finalmente, debe destacarse la limitación de las muestras utilizadas para generalizar los resultados a la totalidad de los extensionistas de los distintos países. A la vez, también debe tenerse presente que aún teniendo muestras cuyos resultados pudieran generalizarse, esto no significaría contar con certezas para afrontar las especificidades de los contextos territoriales e institucionales locales (es decir, subnacionales). De esta manera, se hace evidente que los resultados de este trabajo no deben pensarse como lineamientos estrictos de lo que uno puede encontrarse en cada país, sino como hipótesis con potencialidad para ayudar a prever posibles expectativas y a percibirlas en caso de encontrarlas. Así, el interés de estos resultados parecería estar especialmente en su capacidad para abrir el pensamiento a diferentes posibilidades, las cuales siempre tendrán que ser evaluadas en sus especificidades locales y contextuales.

\section{Referencias}

Ardila, J. (2010). Extensión rural para el desarrollo de la agricultura y la seguridad alimentaria: aspectos conceptuales, situación y una visión de futuro. San José, Costa Rica: IICA.

Bianqui, V., Mathot, M., Vázquez, L., \& Landini, F. (2015). Reflexiones en torno a un campo posible: psicología, extensión y desarrollo rural. En F. Landini (Ed.), Hacia una Psicología Rural Latinoamericana (pp. 251-267). Buenos Aires: CLACSO

Carballo, C. (2002). Extensión y transferencia de tecnología en el sector agrario argentino. Buenos Aires: Universidad de Buenos Aires.

Christoplos, I. (2010). Movilizing the potencial of rural and agricultural extension. Roma: FAO.

Comisión Económica para América Latina y el Caribe - CEPAL, Instituto Interamericano de Cooperación para la Agricultura - IICA, \& Organización de las Naciones Unidas para la Alimentación y la Agricultura - FAO. (2013). Perspectivas de la agricultura y del desarrollo rural en las Américas: una mirada hacia América Latina y el Caribe 2014. San José, Costa Rica: IICA. Extraído de http://www. fao.org/docrep/019/i3702s/i3702s.pdf

Freire, P. (1973). ¿Extensión o comunicación? La concientización en el medio rural. Buenos Aires: Siglo XXI.

Giraldo, M. (2011). Abordaje de la investigación cualitativa a través de la teoría fundamentada en los datos. Ingeniería Industrial. Actualidad y Nuevas Tendencias, 6, 79-86. Extraído de http://www.redalyc.org/ articulo. oa? $\mathrm{id}=215021914006$

Gómez Leyton, J. (2008). Política y ciudadanía en una sociedad neoliberal avanzada, Chile 1990-2007. Cuadernos del Cendes, 25(67), 59-83. Extraído de http://www.scielo.org.ve/scielo. php? script=sci_arttext\&pid=S1012-25082008000100004\&lng=e s\&tlng=es

Hernández Sampieri, R., Fernández Collado, C., \& Baptista Lucio, M. (2010). Metodología de la investigación (5ta ed.). México: McGrawHill.
Instituto Nacional de Tecnología Agropecuaria (2004). El INTA que queremos. Plan Estratégico Institucional 2005 - 2015. Buenos Aires: INTA. Extraído de http://inta.gob.ar/documentos/planestrategico-institucional-2005-2015/at_multi_download/file/Plan\%20 Estrategico\%20INTA\%20\%282005-2015\%29.pdf

Landini, F. (en prensa). Types of rural extensionists' expectations of psychology and their implications on psychologists'. Integrative Psychological and Behavioral Science. doi:10.1007/s12124-016-9341-y

Landini, F. (2012). Problemas en la extensión rural paraguaya: modelos de extensión en la encrucijada. Cuadernos de Desarrollo Rural, 9(69), 127-149. Extraído de http://revistas.javeriana.edu.co/index. $\mathrm{php} /$ desarrolloRural/article/view/4370

Landini, F. (2015a). La noción de psicología rural y sus desafíos en el contexto latinoamericano. En F. Landini (Ed.), Hacia una psicología rural latinoamericana (pp. 21-32). Buenos Aires: CLACSO.

Landini, F. (2015b). Contributions of community psychology to rural advisory services: An analysis of Latin American rural extensionists' point of view. American Journal of Community Psychology, 55(3), 359-368. doi: 10.1007/s10464-015-9712-4.

Landini, F. (2016). Concepción de extensión rural en 10 países latinoamericanos. Manuscrito sometido para publicación. Andamios, 13(30), 211-236. Extraído de http://www.redalyc.org/articulo. oa? $\mathrm{id}=62845862011$

Landini, F., \& Bianqui, V. (2014). Socio-demographic profile of different samples of Latin American rural extensionists. Ciência Rural, 44(3), 575-581. doi: 10.1590/S0103-84782014000300030

Landini, F., Leeuwis, C., Long, N., \& Murtagh, S. (2014). Towards a psychology of rural development processes and interventions. Journal of Community and Applied Social Psychology, 24(6), 534546. doi: 10.1002/casp.2187

Landini, F., Long, N., Leeuwis, C., \& Murtagh, S. (2014). Theoretical guidelines for a psychology of rural development processes and interventions. Cuadernos de Desarrollo Rural, 11(74), 125-147. doi: 10.11144/Javeriana.CRD11-74.tgpr

Landini, F., Murtagh, S., \& Lacanna, M. (2009). Aportes y reflexiones desde la psicología al trabajo de extensión con pequeños productores. Formosa, Argentina: INTA.

Landini, F., \& Riet, L. (2015). Extensión rural en Uruguay: problemas y enfoques vistos por sus extensionistas. Mundo Agrario, 16(32). Extraído de: http://www.mundoagrario.unlp.edu.ar/article/view/ MAv16n32a09/6862

Leeuwis, C. (2004). Communication for rural innovation. Rethinking agricultural extension. Oxford: Blackwell Science.

Leeuwis, C., \& Aarts, N. (2011). Rethinking communication in innovation processes: Creating space for change in complex systems. Journal of Agricultural Education and Extension, 17(1), 21-36. doi: 10.1080/1389224X.2011.536344

Moraes, A., Oreggioni, W., \& Picos, G. (2010). El "Programa de Formación de Actores Locales para el Desarrollo Rural”. En A. Moraes, W. Oreggioni, \& G. Picos (Eds.), Formación para el desarrollo rural (pp. 25-37). Montevideo: Universidad de la República.

Murtagh, S., \& Landini, F. (2011). Producción científica de la psicología vinculada a pequeños productores agropecuarios con énfasis en el ámbito del desarrollo rural. Revista Interamericana de Psicología, 45(2), 293-304. Extraído de https://journal.sipsych.org/index.php/ IJP/article/view/159 
Organización de las Naciones Unidas para la Alimentación y la Agricultura - FAO (2015). The State of food and agriculture 2015. Social protection and agriculture: breaking the cycle of rural poverty. Roma: FAO.

Pérez, C. (2001). Técnicas estadísticas con SPSS. Madrid: Prentice Hall.

Quispe, J., \& Delgado, M. (2010). Modelo comunitario para el desarrollo integral de las comunidades en Bolivia. Ingeniería Industrial, 31(1). Extraído de http://rii.cujae.edu.cu/index.php/revistaind/article/ view/293/307
Sulaiman, R., \& Davis, K. (2012). The "New Extensionist": Roles, strategies, and capacities to strengthen extension and advisory services. Lindau, Suiza: Global Forum for Rural Advisory Services. Extraído de http://www.g-fras.org/en/knowledge/gfras-publications/ file/126-the-new-extensionist-position-paper?start=20

Vakis, R., Rigolini, J., \& Lucchetti, L. (2015). Left behind. Chronic poverty in Latin America and the Caribbean. Washington: Banco Mundial.

${ }^{1}$ Información extraída el 12 de julio de 2016 de sitio web del Banco Mundial: http://data.worldbank.org/

Fernando Landini, Dr. en Psicología por la Universidad de Buenos Aires (UBA), Investigador Adjunto en el Consejo Nacional de Investigaciones Científicas y Técnicas (CONICET), es Profesor Asociado de la Universidad de la Cuenca del Plata (UCP) y Profesor Invitado de la Universidad de Morón (UM). Dirección:

Terrerro 1165, 3A. Buenos Aires (1416). Argentina. Teléfono: +54 11 35305522. Correo electrónico: landini_fer@hotmail.com

Recebido em 14.Jun.15

Revisado em 14.Jul.16 Aceito em 14.Out.16 